NASA/TM-2005-213998

\title{
Assessing Potential Propulsion Breakthroughs
}

Marc G. Millis

Glenn Research Center, Cleveland, Ohio 
Since its founding, NASA has been dedicated to the advancement of aeronautics and space science. The NASA Scientific and Technical Information (STI) Program Office plays a key part in helping NASA maintain this important role.

The NASA STI Program Office is operated by Langley Research Center, the Lead Center for NASA's scientific and technical information. The NASA STI Program Office provides access to the NASA STI Database, the largest collection of aeronautical and space science STI in the world. The Program Office is also NASA's institutional mechanism for disseminating the results of its research and development activities. These results are published by NASA in the NASA STI Report Series, which includes the following report types:

- $\quad$ TECHNICAL PUBLICATION. Reports of completed research or a major significant phase of research that present the results of NASA programs and include extensive data or theoretical analysis. Includes compilations of significant scientific and technical data and information deemed to be of continuing reference value. NASA's counterpart of peerreviewed formal professional papers but has less stringent limitations on manuscript length and extent of graphic presentations.

- TECHNICAL MEMORANDUM. Scientific and technical findings that are preliminary or of specialized interest, e.g., quick release reports, working papers, and bibliographies that contain minimal annotation. Does not contain extensive analysis.

- CONTRACTOR REPORT. Scientific and technical findings by NASA-sponsored contractors and grantees.
- CONFERENCE PUBLICATION. Collected papers from scientific and technical conferences, symposia, seminars, or other meetings sponsored or cosponsored by NASA.

- SPECIAL PUBLICATION. Scientific, technical, or historical information from NASA programs, projects, and missions, often concerned with subjects having substantial public interest.

- TECHNICAL TRANSLATION. Englishlanguage translations of foreign scientific and technical material pertinent to NASA's mission.

Specialized services that complement the STI Program Office's diverse offerings include creating custom thesauri, building customized databases, organizing and publishing research results ... even providing videos.

For more information about the NASA STI Program Office, see the following:

- Access the NASA STI Program Home Page at http://www.sti.nasa.gov

- E-mail your question via the Internet to help@sti.nasa.gov

- Fax your question to the NASA Access Help Desk at 301-621-0134

- Telephone the NASA Access Help Desk at 301-621-0390

- Write to:

NASA Access Help Desk

NASA Center for AeroSpace Information 7121 Standard Drive

Hanover, MD 21076 
NASA/TM-2005-213998

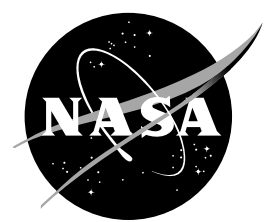

\section{Assessing Potential Propulsion Breakthroughs}

Marc G. Millis

Glenn Research Center, Cleveland, Ohio

Prepared for the

New Trends in Astrodynamics and Applications II-An International Conference sponsored by NASA Headquarters and Princeton University

Princeton, New Jersey, June 3-5, 2005

National Aeronautics and

Space Administration

Glenn Research Center 
Trade names or manufacturers' names are used in this report for identification only. This usage does not constitute an official endorsement, either expressed or implied, by the National Aeronautics and Space Administration.

Available from

NASA Center for Aerospace Information 7121 Standard Drive

Hanover, MD 21076
National Technical Information Service 5285 Port Royal Road Springfield, VA 22100

Available electronically at http:/ /gltrs.grc.nasa.gov 


\title{
Assessing Potential Propulsion Breakthroughs
}

\author{
Marc G. Millis \\ National Aeronautics and Space Administration \\ Glenn Research Center \\ Cleveland, Ohio 44135
}

\begin{abstract}
The term, propulsion breakthrough, refers to concepts like propellantless space drives and fasterthan-light travel, the kind of breakthroughs that would make interstellar exploration practical. Although no such breakthroughs appear imminent, a variety of investigations into these goals have begun. From 1996 to 2002, NASA supported the Breakthrough Propulsion Physics Project to examine physics in the context of breakthrough spaceflight. Three facets of these assessments are now reported: (1) predicting benefits, (2) selecting research, and (3) recent technical progress. Predicting benefits is challenging since the breakthroughs are still only notional concepts, but kinetic energy can serve as a basis for comparison. In terms of kinetic energy, a hypothetical space drive could require many orders of magnitude less energy than a rocket for journeys to our nearest neighboring star. Assessing research options is challenging when the goals are beyond known physics and when the implications of success are profound. To mitigate the challenges, a selection process is described where: (a) research tasks are constrained to only address the immediate unknowns, curious effects or critical issues, (b) reliability of assertions is more important than their implications, and (c) reviewers judge credibility rather than feasibility. The recent findings of a number of tasks, some selected using this process, are discussed. Of the 14 tasks included, 6 reached null conclusions, 4 remain unresolved, and 4 have opportunities for sequels. A dominant theme with the sequels is research about the properties of space, inertial frames, and the quantum vacuum.
\end{abstract}

\section{Introduction}

Confronted by the physical limits of rocketry and space sails, NASA supported the Breakthrough Propulsion Physics Project from 1996 to 2002 [Millis 1999a, 2004a]. As its name suggests, the project specifically looked for propulsion breakthroughs from physics rather than refinements of technology. By breakthroughs, it is meant new propulsion methods that go beyond the limits of rocketry and space sails the kind of breakthroughs that might make human voyages to other star systems possible. Theories and phenomena in recent scientific literature provide new approaches to seek such breakthroughs, including "warp drives" [Alcubierre 1994], wormholes [Visser 1995], vacuum fluctuation energy [Maclay 2004], and emerging physics in general.

This report focuses on the following 3 challenges of this pursuit: (1) predicting benefits, (2) selecting the best research approaches, and (3) the recent technical progress itself. To predict benefits, a number of different assessments are offered. Since little has been published toward quantifying breakthrough benefits, a variety of assessments are offered to set the groundwork for future assessments. The second challenge, that of selecting the best research approaches, is addressed by summarizing the key management strategies from a recent publication about the NASA Breakthrough Propulsion Physics Project [Millis 2004b]. And finally, extracts from recent research findings [Millis 2004a] are compiled with attention drawn to the most immediate research questions.

\section{Predicting Benefits}

Gauging the potential benefits of undiscovered propulsion breakthroughs is challenging, but addressable. The major difficulty is that such breakthroughs are still only notional concepts rather than 
being a specific method from which performance can be unambiguously calculated. One prior assessment considered a Voyager-sized spacecraft using a hypothetical space drive to show that the trip time to reach our nearest neighboring star could be decreased by a factor of 6.5 just by using the leftover power of Voyager's generators [Boston 2000]. Another recent assessment considered a rocket with hypothetical modifications of inertia and gravity and showed that the benefits would be trivial [Tajmar 2005]. Performance estimates can vary considerably depending on the methods and assumptions. To pave the way for a more complete suite of assessments, a variety of methods are introduced here along with a few examples that are worked out. A key feature is that the basis of comparison is energy, rather than using the metrics of rocketry. Discussion on the pitfalls of using rocketry metrics for assessing breakthrough spaceflight is also provided.

\section{Benefits of Hypothetical Modifications of Propellant Inertia}

A recent publication took a first step toward assessing the potential benefits of hypothetical inertial and gravitational control, but did so in terms of rocketry [Tajmar, 2005]. A modified rocket equation was used to demonstrate that naïve modifications of gravity or inertia do not produce much benefit. Although an important first step to help correct misconceptions, this assessment did not include many other relevant comparisons. As an example of a limitation, the published analysis applied a hypothetical inertial change equally to both the propellant and the vehicle. It is not surprising then that there is little or no benefit. One could equally assume that only the inertia of the expelled propellant were increased while the inertia of the vehicle remained the same, in which case there would be more benefit.

The rocket equation is derived next for both a normal rocket and for the case where the expelled propellant's inertia is increased as it is accelerated out of the rocket. The inertial modification is not applied to the rest of the rocket or the stored propellant. It is important to stress that this is only a hypothetical example to illustrate the sensitivity of the findings to the methods, rather than to suggest that this is a realistic potential breakthrough. Numerous variations on this analysis are possible.

The rocket equation is typically derived starting with conservation of momentum, where the momentum of the rocket in one direction must equal the momentum of expelled propellant in the other. For convenience we can set the initial velocity of the rocket to zero. Due to the one-dimensional nature of the problem, vector notation is not needed, but close attention must be paid to the proper sign assignments. Also, the following treatment only represents field-free space where no external forces are acting. Below are the conservation of momentum equations for both the rocket (r) and the modified rocket system (MRS):

The Rocket

$-v_{e} d m=d v_{r}(m-d m)$
The Modified Rocket System (MRS)

$-v_{e}(\delta) d m=d v_{M R S}(m-d m)$

The left sides of both equations represent the momentum of the expelled propellant and the right sides represent the corresponding momentum of the accelerated rockets, and where;

$d m \quad$ incremental mass of expelled propellant

$v_{e} \quad$ exhaust velocity of propellant (opposite to the direction of the rocket motion, hence the negative sign)

$d v_{r} \quad$ incremental change in velocity of the rocket (in the direction of motion, hence the positive sign)

$d v_{M R S}$ incremental change in velocity of the modified rocket system (MRS)

$m \quad$ mass of both rockets (including stored propellant) 
$\delta \quad$ degree of inertia modification, where $\delta=1$ represents no modification, greater than 1 is an increase, and less than 1 is a decrease. Note that this $\delta$ is applied to the propellant as it exits the rocket; hence it acts only on the $d m$ term on the left side of the equation. Delta $(\delta)$ is shown in parenthesis to make it easier to locate in the derivation that follows.

It is standard practice when deriving the rocket equation to approximate that the expelled propellant is trivially small when compared to the mass of the rocket and its stored propellant [Berman 1961, Seifert 1959]. This simplification can be argued in view of a very short time increment for the expulsion. Thus, the $d m$ term on the right-hand side of the equation is a negligible and can be zeroed, while the left-hand $d m$ term still plays a significant role and must remain. Other derivations involving thrust and momentum conversation yield the same results without this approximation [Resnick 1977]. Repeating this analysis using this different derivation is a suitable topic for future work. Applying this $(d m<<m)$ assumption yields:

The Rocket

$$
-v_{e} d m=m d v_{r}
$$

The Modified Rocket System (MRS)

$$
-v_{e}(\delta) d m=m d v_{M R S}
$$

Integrate with respect to mass and velocity:

The Rocket

$$
-v_{e} \int_{m_{i}}^{m_{f}} \frac{1}{m} d m=\int_{v_{i}}^{v_{f}} d v_{r}
$$

The Modified Rocket System (MRS)

$$
-v_{e}(\delta) \int_{m_{i}}^{m_{f}} \frac{1}{m} d m=\int_{v_{i}}^{v_{f}} d v_{M R S}
$$

Where the new terms are;

$\begin{array}{ll}m_{i} & \text { Initial mass of the rockets (before expulsion of propellant) } \\ m_{f} & \text { Final mass of the rockets (after expulsion of propellant) } \\ v_{i} & \text { Initial velocity of the rockets } \\ v_{f} & \text { Final velocity of the rockets }\end{array}$

Calculating the integrals yields:

The Rocket

$$
-v_{e}\left[\ln \left(m_{f}\right)-\ln \left(m_{i}\right)\right]=v_{f}-v_{i}=\Delta v_{r} \quad-v_{e}(\delta)\left[\ln \left(m_{f}\right)-\ln \left(m_{i}\right)\right]=v_{f}-v_{i}=\Delta v_{M R S}
$$

Note that the changes in velocity of the rockets are now represented as $\Delta v^{\prime} s$. Distributing the sign and applying a logarithmic identity yields the celebrated Tsiolkovski equation of 1903 [Berman 1961] on the left, and a slightly modified version of the same on the right for the Modified Rocket System:

The Tsiolkovski Rocket Equation

$$
\Delta v_{r}=v_{e} \ln \left(\frac{m_{i}}{m_{f}}\right)
$$

The Modified Rocket System Equation (MRS)

$$
\Delta v_{M R S}=(\delta) v_{e} \ln \left(\frac{m_{i}}{m_{f}}\right)
$$


Finally, to compare the performance improvement of the Modified Rocket System with the rocket, take the ratio of their $\Delta v$ 's and require that all other values are identical:

$$
\frac{\Delta v_{M R S}}{\Delta v_{r}}=\frac{v_{e}(\delta) \ln \left(\frac{m_{i}}{m_{f}}\right)}{v_{e} \ln \left(\frac{m_{i}}{m_{f}}\right)}
$$

Reducing this yields:

$$
\frac{\Delta v_{M R S}}{\Delta v_{r}}=\delta
$$

This means that a delta of 1.10 , representing a 10 percent increase in the expelled propellant's inertia, would yield a 10 percent increase in $\Delta v$. While this appears encouraging, it should be remarked that there are at present no known techniques to affect such a change in propellant inertia and the result is only meant to illustrate a potential advantage that hypothetical inertial modifications might provide. A separate issue to pursue would be to calculate the energy required to support this hypothetical change in propellant inertia. Again, the main point of the exercise is to reveal that different approaches will yield significantly different results. The implications of Equation (1.7) are considerably different than the null finding which occurs when one applies the inertial modification to both sides of the equation [Tajmar 2005].

\section{Limits of Rocketry Analyses for Space Drives}

When using the metrics of an incumbent technology to assess the potential of a new technology, results can be misleading. The example above is just one illustration of how two different assumptions of hypothetical inertial control via a rocket equation can lead to very different answers. Another misleading use of the rocket equation when contemplating breakthroughs is in the common practice of assigning an infinite specific impulse to describe a propellantless space drive. Although based on a reasonable extrapolation of the trend where higher specific impulse leads to less required propellant, this also leads to the conclusion that a propellantless space drive would require infinite energy. As shown later, this is not necessarily the case. Furthermore, since specific impulse is a measure of the thrust per propellant weight flow rate, it has no real meaning if there is no propellant flow.

Using the rocket equation to describe something that is not likely to involve a rocket is about as misleading as using the metrics of sailing ships to assess steamships [analogy from Foster 1986]. Although reduced sails and rigging is indeed a consequence of steamships, the true benefit is that shipping can continue regardless of the wind conditions and with far more maneuvering control. Similarly, the benefits of breakthrough inertial or gravity control would likely surpass the operational conventions of rocketry. Although comparisons built on the incumbent methods might be useful for introductory purposes, a deeper understanding of the benefits and research approaches are better illustrated by using a common and more basic metric. For spaceflight, whether via rockets or space drives, energy is a better basis for comparison.

\section{Specific Impulse Limits}

Before proceeding to demonstrate the use of energy as a basis for comparison, a limit should be brought to attention. For these introductory exercises, the comparisons are limited to non-relativistic regimes. For rockets, this implies limiting the exhaust velocity to $\leq 10$ percent lightspeed. The 
corresponding upper limit to specific impulse easily follows from the equation relating specific impulse to exhaust velocity [Seifert 1959]:

$$
v_{e}=I_{s p} g
$$

Where

$I_{s p}$ "Specific Impulse" (sec) which is a measure of the rocket's propellant efficiency, specifically the amount of thrust per propellant weight flow rate.

$g \quad$ Earth's gravitational field $=9.8 \mathrm{~m} / \mathrm{s}^{2}$.

Setting the exhaust velocity of 10 percent light-speed (beyond which relativistic effects must be considered), the limiting specific impulse is found to be:

$$
(10 \text { percent })\left(3.0 \times 10^{8} \frac{m}{s}\right) \geq I_{s p}\left(9.8 \frac{m}{s^{2}}\right) \Rightarrow I_{s p} \leq 3.0 \times 10^{6} s
$$

\section{Energy Comparisons for Deep Space Travel}

This next assessment deals with deep space travel. In this section, both a rocket and a hypothetical space drive will be compared in terms of energy requirements. A space drive is defined as: "an idealized form of propulsion where the fundamental properties of matter and spacetime are used to create propulsive forces anywhere in space without having to carry and expel a reaction mass" [Millis 1997]. For this exercise it can be thought simply as a device that converts potential energy directly into kinetic energy. Since issues such as momentum conservation are addressed in the cited reference, they will not be elaborated on here.

\section{Assumptions}

For this introductory exercise, the following assumptions are used. To more fully understand the challenges, approaches and potential benefits of breakthrough propulsion, it would be fruitful to repeat the analysis using different assumptions:

Both the rocket and the space drive are assumed to be 100 percent efficient with their energy conversions.

The thrusting duration is assumed to be much shorter than the trip duration, which for interstellar travel is reasonable.

For the rocket, constant exhaust velocity is assumed.

Non-relativistic trip velocity and exhaust velocity are assumed.

The energy requirements for a rendezvous mission are based on equal $\Delta v$ 's for acceleration and deceleration.

\section{Energy of a Rocket}

To compare the energy of a rocket to another method that does not require propellant, we need an equation for the kinetic energy where the propellant mass is represented in terms of the vehicle's empty mass and the $\Delta v$ of the mission. A common way to calculate the total kinetic energy of a rocket system, including both the rocket and the propellant, is just to calculate the energy imparted to the propellant from the rocket's frame of reference where the rocket has zero velocity (hence zero kinetic energy) [Berman 1961 and Seifert 1959]. This is consistent with the previously stated assumptions. 


$$
\Delta E=\frac{1}{2}\left(m_{i}-m_{\text {empty }}\right) v_{e}^{2}
$$

Where the parenthetical term is the propellant mass and:

$m_{\text {empty }}$ mass of the vehicle without the propellant, which will be the same value used for the space drive.

$m_{i} \quad$ initial mass of the vehicle before the expulsion of propellant.

$v_{e} \quad$ exhaust velocity of the rocket.

Next, to convert this into a form where the rocket's propellant mass $\left(m_{i}-m_{\text {empty }}\right)$ is represented in terms of the exhaust velocity and the mission $\Delta v$, we apply the following form of the rocket equation, which is a variation of the Tsiolkovski equation previously derived:

$$
m_{\text {empty }}\left(e^{\left(\frac{\Delta v}{v_{e}}\right)}-1\right)=\left(m_{i}-m_{\text {empty }}\right)
$$

Where the $\Delta v$ is the required change in velocity for the mission. For a rendezvous mission, the $\Delta v$ is twice that of just a flyby mission. Substituting this form of the rocket equation into the kinetic energy equation yields this very simple approximation:

$$
\Delta E=\frac{1}{2}\left(v_{e}\right)^{2} m_{\text {empty }}\left(e^{\left(\frac{\Delta v}{v_{e}}\right)}-1\right)
$$

\section{Energy for a Space Drive}

Since a space drive has been defined for this exercise as a device that converts potential energy into kinetic energy, the basic equation of kinetic energy is used to calculate the energy requirement, where the values of vehicle mass and mission $\Delta v$ are the same as with the rocket.

$$
\Delta E=\frac{1}{2} m_{\text {empty }}(\Delta v)^{2}
$$

\section{Comparisons}

Two things are important to note regarding the energy differences between a rocket and a hypothetical space drive. First, the energy for a given $\Delta v$ scales as an exponent for a rocket and scales as the square of the $\Delta v$ for a space drive. This by itself is significant, but it is important to point out that a rocket and a space drive treat additional maneuvers differently. For a rocket it is conventional to talk in terms of increases to $\Delta v$ for additional maneuvers. For space drives, however, the additional maneuvers are in terms of additional kinetic energy. To illustrate this difference, consider a mission consisting of multiple maneuvers $(\boldsymbol{n})$ each having the same incremental change in velocity $\left(\Delta v_{i}\right)$. Notice the location of the term representing the number $(\boldsymbol{n})$ of repeated maneuvers $\left(\Delta v_{i}\right)$, in the following two equations. In the case of the space drive, additional maneuvers scale linearly, while for rockets they scale exponentially. This is another example to highlight why rocket conventions can be misleading when contemplating space drives: 


\section{Rocket Maneuvers}

$$
\Delta E=\frac{1}{2}\left(v_{e}\right)^{2} m_{\text {empty }}\left(e^{\left((\boldsymbol{n}) \frac{\Delta v_{i}}{v_{e}}\right)}-1\right)
$$

Space Drive Maneuvers

$$
\Delta E=(\boldsymbol{n}) \frac{1}{2} m_{\text {empty }}\left(\Delta v_{i}\right)^{2}
$$

\section{Numerical Example}

To put this into perspective, consider a representative mission of sending a $5000 \mathrm{~kg}$ probe over a distance of 5 light-years in a 50-year timeframe. This range is representative of the distance to our nearest neighboring star (4.3 light-years) and the 50-yr time frame is chosen as one short enough to be within the threshold of a human career span, yet long enough to be treated with non-relativistic equations. This equates to a required trip velocity of 10 percent lightspeed. The probe size of $5000 \mathrm{~kg}$ is roughly that of the Voyager probe plus the dry mass of the Centaur Upper Stage $(4075 \mathrm{~kg})$ that propelled it out of Earth's orbit [Boston 2000]. The comparison is made for both a flyby mission and a rendezvous mission.

The rocket case is calculated for two different specific impulses, one set at the upper non-relativistic limit previously described, and another set at a high value achieved during electric propulsion lab tests [Byers 1969]. The Space Drive Improvement column is the ratio of the rocket energy to the space drive energy.

\begin{tabular}{|c|c|c|c|c|}
\hline & Specific Impulse & Mission & Joules Required & $\begin{array}{l}\text { Space Drive } \\
\text { Improvement }\end{array}$ \\
\hline $\begin{array}{l}\text { Rockets } \\
\text { Upper Lab Limit [Byers 69] }\end{array}$ & $17,200 \mathrm{sec}$ & $\begin{array}{l}\text { Flyby } \\
\text { Rendezvous }\end{array}$ & $\begin{array}{l}10^{91} \\
10^{168}\end{array}$ & $\begin{array}{l}10^{72} \\
1^{149}\end{array}$ \\
\hline Non-Relativistic Upper Limit & $3,000,000 \mathrm{sec}$ & $\begin{array}{l}\text { Flyby } \\
\text { Rendezvous }\end{array}$ & $\begin{array}{l}3.8 \times 10^{18} \\
\mathbf{1 . 5} \times \mathbf{1 0}^{19}\end{array}$ & $\begin{array}{l}1.7 \\
3.2\end{array}$ \\
\hline Space Drive & $\begin{array}{l}\text { N/A } \\
\text { N/A } \\
\end{array}$ & $\begin{array}{l}\text { Flyby } \\
\text { Rendezvous }\end{array}$ & $\begin{array}{l}2.3 \times 10^{18} \\
4.5 \times 10^{18}\end{array}$ & \\
\hline
\end{tabular}

TABLE I.-COMPARISON OF DEEP SPACE MISSION ENERGY REQUIREMENTS

Even in the case of the non-relativistic upper limit to specific impulse - an incredibly highperformance hypothetical rocket - the space drive uses a factor of 2 to 3 less energy. When compared to attainable values of specific impulse - values that are still considerably higher than that currently used in practice - the benefits of a space drive are enormous. Even for just a flyby mission, the gain is 72 orders of magnitude. When considering a rendezvous mission, the gain is almost 150 orders of magnitude better. Again, though these results are intriguing, they should only be interpreted as the magnitude of gains sought by breakthrough propulsion research. Further variations of such assessments are possible.

\section{Energy Comparisons for Earth to Orbit}

Consider the case of lifting an object off the surface of the Earth and placing it into orbit. This requires energy expenditures both for the altitude change and for the speed difference between the Earth's surface and the orbital velocity. For the hypothetical space drive, this energy expenditure can be represented as:

$$
E_{\text {SpaceDrive }}=\Delta U+\Delta K
$$


Where $\Delta U$ is the potential energy change associated with the altitude change, and $\Delta K$ is the kinetic energy change associated with different speeds at the Earth's surface and at orbit. First the change in potential energy, which requires expending work to raise a mass in a gravitational field:

$$
\Delta U=\int_{\text {Surface }}^{\text {Orbit }} F \cdot d r=\int_{r_{\text {Sufface }}}^{r_{\text {orbit }}} G \frac{M_{\text {Earth }}}{r^{2}} m_{\text {craft }} d r
$$

Where:

$$
\begin{array}{ll}
F & \text { Force due to gravity } \\
G & \text { Newton's gravitational constant } \\
M_{\text {Earth }} & \text { mass of the Earth } \\
m_{\text {craft }} & \text { mass of the spacecraft } \\
r & \text { radius from the center of the Earth } \\
r_{\text {Orbit }} & \text { radius of the orbit as measured from the center of the Earth } \\
r_{\text {Surface }} & \text { radius of the Earth's surface }
\end{array}
$$

As a comparative example, we can calculate the energy required by a 100 percent efficient hypothetical space drive to launch the mass of the Space Shuttle into orbit. Only the Orbiter mass $\left(9.76 \times 10^{4} \mathrm{~kg}\right)$ is used for these comparative assessments instead of the rest of the Shuttle system elements.

$$
\Delta U=\left(6.67 \times 10^{-11} \frac{\mathrm{m}^{3}}{\mathrm{~kg} \cdot \mathrm{s}^{2}}\right)\left(5.98 \times 10^{24} \mathrm{~kg}\right)\left(9.76 \times 10^{4} \mathrm{~kg}\right)\left(\frac{1}{6.37 \times 10^{6} \mathrm{~m}}-\frac{1}{6.67 \times 10^{6} \mathrm{~m}}\right)=2.75 \times 10^{11} \mathrm{Joules}
$$

The change in kinetic energy requires solving for the orbital velocity and the velocity of the Earth's surface, as shown by:

$$
\Delta K=K_{\text {Orbit }}-K_{\text {Surface }}=\frac{1}{2} m_{\text {craft }}\left(v_{\text {Orbit }}^{2}-v_{\text {Surface }}^{2}\right)=\frac{1}{2} m_{\text {craft }}\left(\left[G \frac{M_{\text {Earth }}}{r_{\text {Orbit }}}\right]-\left[\frac{2 \pi r_{\text {Earth }}}{1 \text { day }}\right]^{2}\right)
$$

Where the additional terms are:

$K_{\text {Orbit }} \quad$ Kinetic energy of the Shuttle Orbiter in orbit

$K_{\text {Surface }}$ Kinetic energy of the Shuttle Orbiter when at rest relative to the Earth's surface, but where the Earth's surface is rotating

$v_{\text {Orbit }} \quad$ Orbital speed

$v_{\text {Surface }}$ Speed of the Earth's surface due to its daily rotation

Again, for the case of a hypothetical space drive placing the mass of the Space Shuttle into Low Earth Orbit (LEO), the following values apply:

$$
\Delta K=\frac{1}{2}\left(9.76 \times 10^{4} \mathrm{~kg}\right)\left(\left[\left(6.67 \times 10^{-11} \frac{\mathrm{m}^{3}}{\mathrm{~kg} \cdot \mathrm{s}^{2}}\right) \frac{5.98 \times 10^{24} \mathrm{~kg}}{6.67 \times 10^{6} \mathrm{~m}}\right]-\left[\frac{2 \pi\left(6.37 \times 10^{6} \mathrm{~m}\right)}{86400 \mathrm{~s}}\right]^{2}\right)=2.91 \times 10^{12} \text { Joules }
$$


Combining the changes in potential and kinetic energy gives the total energy required for a hypothetical space drive to put the mass of the Space Shuttle Orbiter into Low Earth Orbit:

$$
E_{\text {SpaceDrive }}=3.18 \times 10^{12} \text { Joules }
$$

To assess the required energy for the rocket, the actual values of the Space Shuttle System (STS) are used, values extracted from "STS-3 Thirds Space Shuttle Mission Press Kit, March 82," Release no. 82 to 29. In this case the rocket power equation is employed and energy is calculated from the product of the rocket power and thrusting duration. The shift to describing rocket performance in terms of power is done for two reasons: first to highlight this form of the rocket equation [Berman 1961] and secondly to introduce the idea of contemplating power in addition to just energy. While power implications are not explored here in detail, they constitute a fertile area for further study.

Starting with the equation for rocket power [Berman 1961]:

$$
P=\frac{1}{2} F I_{s p} g
$$

Where the new terms are:

\section{$P \quad$ Power \\ $F \quad$ Rocket thrust force}

Applying the physics relation where energy is the product of power and time, yields the following equation for rocket energy in terms of power and thrusting duration:

$$
E=\frac{1}{2} F I_{s p} g t
$$

Where the new term is:

\section{$t \quad$ Thrusting duration}

There are three different types of rockets that make up the STS: the Space Shuttle Main Engines (SSME), the Solid Rocket Boosters (SRB), and the Orbital Maneuvering System (OMS). The total energy is the combination of the energy of each of these:

$$
E_{S T S}=E_{S S M E}+E_{S R B}+E_{O M S}
$$

Where the energy for each of these rockets is:

$$
\begin{aligned}
& E_{S S M E}=(3 \text { engines }) \frac{1}{2}\left(470 \times 10^{3} \text { lbsThrust / engine }\right)\left(4.45 \frac{N}{l b}\right)\left(453 s I_{s p}\right)\left(9.8 \frac{m}{s^{2}}\right)(514 s \text { BurnDuration })=7.17 \times 10^{12} \text { Joules } \\
& E_{S R B}=(2 \text { boosters }) \frac{1}{2}\left(2.9 \times 10^{6} \text { lbsThrust } / \text { booster }\right)\left(4.45 \frac{\mathrm{N}}{l b}\right)\left(266 s I_{s p}\right)\left(9.8 \frac{\mathrm{m}}{\mathrm{s}^{2}}\right)(126 \mathrm{~s} \text { BurnDuration })=4.24 \times 10^{12} \text { Joules } \\
& E_{\text {OMS }}=(2 \text { engines }) \frac{1}{2}\left(6 \times 10^{3} \text { lbsThrust / engine }\right)\left(4.45 \frac{\mathrm{N}}{\mathrm{lb}}\right)\left(313 s I_{s p}\right)\left(9.8 \frac{\mathrm{m}}{\mathrm{s}^{2}}\right)(200 \mathrm{~s} \text { BurnDuration })=1.64 \times 10^{10} \text { Joules }
\end{aligned}
$$


Combining these values yields:

$$
E_{S T S}=1.14 \times 10^{13} \text { Joules }
$$

Comparing this rocket energy value to the hypothetical space drive energy, where the efficiency of both systems is assumed to be 100 percent, yields:

$$
\frac{E_{\text {STS }}}{E_{\text {SpaceDrive }}}=\frac{1.14 \times 10^{13} \text { Joules }}{3.18 \times 10^{12} \text { Joules }}=3.58
$$

This means that a hypothetical space drive is potentially more than 3-and-a-half times more energy efficient. When compared to the benefits of interstellar space drives, however, this gain is small. From these cursory analyses, space drives do not appear as attractive for launching spacecraft into low orbit as they do for high $\Delta v$ missions or missions that require many maneuvers. Again, such introductory comparisons should not be taken too literally. These assessments are provided to demonstrate that there are a variety of ways to assess the potential benefits of propulsion breakthroughs.

\section{Energy for Levitation}

Levitation is included here mostly to illustrate how contemplating breakthrough propulsion is different from contemplating rocketry. Rockets can hover, but not for very long before they run out of propellant. For an ideal breakthrough, some form of indefinite levitation is desirable, but there is no clear way how to represent the energy or power to perform this feat. Since physics defines work (energy) as the product of force acting over distance, no work is performed if there is no change in distance. Levitation means hovering with no change in height. Regardless, there are a variety of ways to toy with the notion of energy and power for indefinite levitation. A few of these approaches are listed in the next session. For now, only one approach is illustrated, specifically the nullification of gravitational potential.

An object in a gravitational field has the following defined value for its gravitational potential energy:

$$
E=m g h=m \frac{G M_{\text {Earth }}}{h}
$$

Where:

$\begin{array}{ll}m & \text { mass of the object } \\ g & \text { acceleration due to gravity } \\ h & \text { levitation height as measured from the center of the Earth } \\ G & \text { Newton's gravitational constant } \\ M_{\text {Earth }} & \text { mass of the Earth }\end{array}$

Usually this potential energy is used to compare energy differences between two relatively short differences in height $(h)$ but in our situation we are considering this potential energy in the more absolute sense. This same value of potential energy can also be determined by calculating how much energy it would take to completely remove the object from the gravitational field, as if moving it to infinity. This is more in line with the analogy to nullify the effect of gravitational energy. This is also the same amount of energy that is required to stop an object at the levitation height $(h)$ if it were falling in from infinity with an initial velocity of zero. To illustrate this equivalence of these treatments, start with the equation used 
earlier to calculate the change in potential energy for entering orbit, but this time use different limits of integration:

$$
E=\int_{\text {LevitationHeight }}^{\text {Infinity }} F \cdot d r=\int_{h}^{\infty} G \frac{m M_{\text {Earth }}}{r^{2}} d r=m G M_{\text {Earth }}\left[\frac{1}{h}-\frac{1}{\infty}\right]=m \frac{G M_{\text {Earth }}}{h}=m g h(4
$$

It is clear that this is identical to the gravitational potential energy in Equation (4.1). Using the analogy of removing the effect of gravitational potential energy, it is reasonable to conjecture that this is one way to calculate the amount of energy required to permanently levitate an object. Inserting values representative of levitating 1-kg near the Earth's surface yields:

$$
E=1 \mathrm{~kg}\left(9.80 \frac{\mathrm{m}}{\mathrm{s}^{2}}\right) 6.37 \times 10^{6} \mathrm{~m}=62 \mathrm{M} \text { Joules }
$$

Hence, to levitate 1-kg near the Earth's surface could conceivably require 62 Mega-Joules. This is roughly twice as much as putting 1-kg into Low Earth Orbit. Again, these assessments are strictly for illustrative purposes rather than suggesting that such breakthroughs are achievable or if they would even take this form if achievable. Some starting point for comparisons is needed, and this is just one version.

\section{Assessing Benefits More Generally}

As illustrated with these introductory examples, there are a number of different ways to assess the potential benefits of breakthrough physics propulsion. To continue with deeper inquiry, a variety of missions and assumptions can be addressed. The following list is just a starting point for further analyses. Those items marked in bold font are the ones already introduced in this paper.

1. Deep space travel (motion from point A to B without external forces):

a. Rocketry baselines:

i. Non-relativistic Energy (velocity less than 10 percent lightspeed):

\section{Constant exhaust velocity and short thrust durations.}

2. Constant thrust.

3. Constant acceleration.

4. Optimized for minimum trip times.

ii. Relativistic Energy (cases 1 to 4 above repeated with relativistic corrections).

b. Space Drive motion using mechanical analogies:

i. Non-relativistic Energy:

1. Simple kinetic energy differences.

2. Kinetic energy under constant acceleration.

3. Kinetic energy under constant power.

ii. Relativistic Energy (cases 1 to 3 above repeated with relativistic corrections).

c. Space Drive motion using geometric spacetime analyses:

i. Creating a pseudo geodesic — reshaping spacetime to induce the preferred freefall trajectory.

ii. Warp Drive - moving a chunk of spacetime [Alcubierre 1994].

iii. Wormhole - moving through a shortcut in spacetime [Visser 1995].

iv. Krasnikov tube - creating a faster-than-light geodesic [Krasnikov 1998].

2. Ascent to orbit (motion in a gravitational field with the destination being a stable orbit):

a. Rocketry ascent baselines:

i. Space Shuttle System data.

ii. Generic staged rocket ascent. 
b. Space Drive ascent using mechanical analogies:

i. Simple kinetic and potential energy differences using Space Shuttle data.

ii. Ascent under constant power.

3. Levitating in a gravitational field:

a. Rocketry levitation baseline: Levitation duration at the Earth's surface.

b. Space Drive levitation using mechanical analogies;

i. Normal physics definition of work, where zero change in height equates to zero energy expenditure.

ii. Comparison to continual down thrust of a reaction mass (rocket and helicopter analogy).

iii. Comparison to normal accelerated motion in free space, where distance is traversed.

iv. By negating gravitational potential, as if moving a mass to infinity.

v. Comparing to kinetic energy associated with escape velocity.

vi. Thermodynamic approach: Seeking equations for the energy and power to keep a system in a stationary state away from its equilibrium condition, where the equilibrium condition is free-fall motion of a mass in a gravitational field and the stable non-equilibrium condition is levitation at a given height.

vii. Assuming a "gravity shield," but for illustrative purposes consider it located under half of a vertical wheel to calculate the energy associated with the increasing rotation rate of the wheel.

viii. Calculating the energy of oscillation about an median hovering height, but where an energy cost is incurred for both the upward and downward excursions, and where damping losses are included.

ix. Analyze using the "impulse" treatment (force $\mathrm{x}$ duration, rather than force $\mathrm{x}$ distance).

c. Space Drive levitation in terms of geometric general relativity-inducing a null geodesic where the local freefall path is a stationary trajectory.

\section{Selecting Research Approaches}

A normal challenge of any research project is directing limited resources to the best prospects. The hunt for incredible breakthroughs faces the additional challenge of making credible progress. Because the desired propulsion breakthroughs are presumably far from fruition and provocative, specific strategies were devised in the course of the NASA Breakthrough Propulsion Physics (BPP) Project to mitigate the risks and maximize progress [Millis 2004b]. The BPP Project employed the operating strategies described below. Other details, such as the specific selection criteria, evaluations equations, review process, and lessons learned, are presented in Millis [2004b].

\section{Reliability}

Although it is a common practice when advocating research to emphasize the ultimate technical benefits, this practice is not constructive on topics as visionary and provocative as breakthrough spaceflight. Instead, it is more constructive to emphasize the reliability of the information being offered. Compared to other propulsion research, new propulsion physics is at its infancy. It is expected, therefore, that any practical embodiment is years, perhaps decades away, if not impossible. Although breakthroughs, by their very definition, happen sooner than expected, no breakthrough is genuine until it has been proven to be genuine. Hence, the reliability of the information is a paramount prerequisite to the validity of any conclusions. To place the emphasis where it is needed, no research approach should be considered unless credibility is satisfactorily addressed, regardless of the magnitude of claimed benefit. Success is defined as acquiring reliable knowledge, rather than as achieving a breakthrough. 


\section{Immediacy}

Another technique to shift the emphasis away from provocative situations and toward constructive practices is to focus the research on the immediate questions at hand. These immediate unknowns, issues, and curious effects can be identified by comparing established and emerging physics to the ultimate propulsion goals. The scope of any research task should ideally be set to the minimum level of effort needed to resolve an immediate "go/no-go" decision on a particular approach. This near-term focus for long-range research also makes the tasks more manageable and more affordable. Specifically, it is recommended that any proposed research be configured to reach a reliable conclusion in one to three years. Should the results be promising, a sequel can be proposed in the next solicitation cycle.

\section{Measured}

To help identify a suitable research increment and to provide managers a means to measure progress, the Scientific Method can be adapted as a readiness scale in a manner similar to how the Technology Readiness Levels are used to measure technological progress [Hord 1985]. The readiness scale developed for the BPP Project consists of three stages that gauge the applicability of the work (reflecting how research can evolve from the more general, to the more specific application), and within each of these 3 stages, the 5 steps of the scientific method are repeated (from recognizing the problem, through testing the hypothesis). This equates to 15 levels of relative maturity, with the most advanced level being equivalent to Technology Readiness Level 1 (Basic principles observed and reported). An abbreviated version of these "Applied Science Readiness Levels" is presented in Table II, and further details are available in Millis [2004b]. Once a research objective has been ranked relative to this scale, the next logical increment of research would be to advance that topic to the next higher readiness level. This is consistent with the incremental research strategy.

TABLE II.-APPLIED SCIENCE READINESS LEVELS

\begin{tabular}{|c|l|}
\hline \multicolumn{2}{|l|}{ General Physics-deals with general underlying physics related to the application. } \\
\hline SRL-1.0 & Pre-science (Unconfirmed effect or new information connection) \\
\hline SRL-1.1 & Problem formulated \\
\hline SRL-1.2 & Data collected \\
\hline SRL-1.3 & Hypothesis proposed \\
\hline SRL-1.4 & Hypothesis tested \& results reported \\
\hline $\begin{array}{l}\text { Critical Issues- deals with an immediate unknown, critical make-or-break issue, or curious effect relevant } \\
\text { to the application. }\end{array}$ \\
\hline SRL-2.0 & Pre-science (Unconfirmed effect or new information connection) \\
\hline SRL-2.1 & Problem formulated \\
\hline SRL-2.2 & Data collected \\
\hline SRL-2.3 & Hypothesis proposed \\
\hline SRL-2.4 & Hypothesis tested \& results reported \\
\hline $\begin{array}{l}\text { Desired Effect- deals directly with the effect required by the application (e.g. inducing forces or } \\
\text { generating energy in the case of breakthrough propulsion applications) }\end{array}$ \\
\hline SRL-3.0 & Pre-science (Unconfirmed effect or new information connection) \\
\hline SRL-3.1 & Problem formulated \\
\hline SRL-3.2 & Data collected \\
\hline SRL-3.3 & Hypothesis proposed \\
\hline SRL-3.4 & $\begin{array}{l}\text { Hypothesis } \text { empirically tested \& results reported } \\
\text { (Equivalent to TRL 1: Basic principles observed and reported) }\end{array}$ \\
\hline
\end{tabular}




\section{Iterated}

To accumulate progress over the long term, it is recommended to solicit a suite of proposals every two to three years, and to let the findings of the prior suite influence the next round of selections. This provides an opportunity for new approaches, sequels to the positive results, and redirections around null results. At any point, if a research task leads to the discovery of a new propulsion or energy effect, it can be pulled out of this process into its own advancement plan. This strategic approach is recommended for high-gain/high-risk research, where cycles of peer-reviewed solicitations can examine a diverse portfolio of options, and where the decisions build on the lessons learned from prior cycles of research.

\section{Diversified}

It is far too soon, in the course of seeking spaceflight breakthroughs, to down-select to just one or two hot topics. Instead a variety of research approaches should be investigated in each review cycle. In simple terms, this is to diversify the research portfolio. This is different than the more common practice with advanced propulsion research where further advancements are primarily sought on the technical approaches already under study. Although this more common strategy can produce advances on the chosen topics, it faces the risk of overlooking emerging alternatives and the risk that support will wane unless the chosen topics produce unambiguous positive results.

\section{Impartial}

When inviting research the edge of knowledge, controversial ideas are encountered. Considering that most historic breakthroughs originally sounded like fringe ideas, it is not surprising that many of the proposals for breakthrough spaceflight might sound too visionary at first, or at least unfamiliar. It is therefore difficult to sort out the fringe ideas that may one day evolve into tomorrow's breakthroughs from the more numerous, erroneous fringe ideas. During proposal reviews, it is common to have some reviewers reflexively assume that unfamiliar ideas will not work. To reliably determine technical feasibility, however, is beyond the scope of a proposal review - constituting a full research task unto itself. Instead of expecting proposal reviewers to judge technical feasibility, it is recommended to have reviewers judge if the task is leading to a result that other researchers will consider as a reliable conclusion on which to base future investigations. This includes both the possibility of determining which approaches are nonviable as well as which are candidates for deeper inquiry. This posture of judging credibility rather than pre-judging feasibility is one of the ways of being open to visionary concepts while still sustaining credibility.

\section{Empirical}

When seeking advancements that can eventually lead to new technology, there is a decided preference toward tangible observations over purely analytical studies - all other factors being equal, such as cost, technical maturity, etc. Experiments, being hardware, are considered closer than theory to becoming technology. Also, experiments are considered a more direct indicator of how nature works. Theories are interpretations to explain observations of nature, while the empirical data is nature, partially revealed within the constraints of the given experiment.

\section{Published}

The final recommendation to mitigate the risks of pursuing visionary, high-gain research is to ensure

that the research findings are published, regardless of outcome. Results, pro or con, set the foundations for guiding the next research directions. Although there can be a reluctance to publish null results-where a 
given approach is found not to work - such dissemination will prevent other researchers from repeatedly following dead-ends.

\section{Recent Technical Progress}

The findings of over a dozen separate research tasks related to breakthrough propulsion physics were recently published [Millis 2004a]. These findings are rearranged here according to which tasks proved non-viable, which remain unresolved, and which are candidates for further research. Under each of these headings, the different approaches are only briefly described, but pertinent reference citations are offered for follow-up inquiries.

It should be stressed that even interim positive results do not imply that a breakthrough is inevitable. Often the opportunity for sequels is more a reflection of the embryonic state of the research. Reciprocally, a dead-end conclusion on a given task does not imply that broader topics are equally defunct. Both the null and positive results should be strictly interpreted within the context of the immediate research task. This is consistent with the operating strategy to focus on the immediate stage of the research, and the strategy to put a higher priority on the reliability of the information rather than on producing broadsweeping claims.

It should also be stressed that these task summaries do not reflect a comprehensive list. It is expected that new concepts will continue to emerge in such an embryonic field and that further, more applicable references may already be in the open literature.

\section{Non-Viable Approaches}

\section{Oscillation Thrusters and Gyroscopic Antigravity}

A large number of mechanical devices that claim to produce net external thrust, using just the motion of internal components, are often proposed. These devices fall into two categories, oscillation thrusters and gyroscopic devices. Their appearance of creating net thrust is attributable to misinterpretations of normal mechanical effects. The following short explanations were excerpted and edited from a NASA website about commonly submitted erroneous breakthroughs [Thomas 2002].

Oscillation thrusters move a system of internal masses through a cycle where the motion in one direction is quicker than in the return direction. When the masses are accelerated quickly, the device has enough reaction force to overcome the friction of the floor and the device slides. When the internal masses return slowly in the other direction, the reaction forces are not sufficient to overcome the friction to move the device. The net effect is that the device moves in one direction across a frictional surface. In a frictionless environment the whole system would simply oscillate around its center of mass.

A gyroscopic thruster consists of a system of gyroscopes connected to a central body. When the central body is torqued, the gyros move in a way that appears to defy gravity. Actually the motion is due to gyroscopic precession and the forces are torques around the axes of the gyros' mounts. There is no net thrust created by the system.

To keep an open, yet rigorous, mind to the possibility that there has been some overlooked physical phenomena with such devices, it would be necessary to explicitly address all the conventional objections and pass at least a pendulum test. Any test results would have to be impartial and rigorously address all possible causes that might lead to a false-positive conclusion. There has not yet been any viable theory or experiment that reliably demonstrates that a genuine, external, net thrust can be obtained with one of these devices. If such tests are ever produced, and if a genuine new effect is found, then science will have to be revised, because it would then appear that such devices are violating Conservation of Momentum. 


\section{Hooper Antigravity Coils}

Experiments were conducted to test assertions from US Patent 3,610,971, by W.J. Hooper that selfcanceling electromagnetic coils can reduce the weight of objects placed underneath. No weight changes were observed within the detectability of the instrumentation. More careful examination led to the conclusion that Hooper may have misinterpreted thermal effects as his "Motional Field" effects [Millis 1995].

\section{Schlicher Thrusting Antenna}

Tests of a specially terminated coax, that was claimed to create more thrust than attributable to photon radiation pressure, revealed that no such thrust was present [Fralick 2001].

\section{Podkletnov Gravity Shield}

A controversial claim of "gravity shielding" using rotating superconductors and radio-frequency radiation was published based on work done at Finland's Tampere Institute [Podkletnov 1992]. A privately funded replication of the Podkletnov configuration "found no evidence of a gravity-like force to the limits of the apparatus sensitivity," where the sensitivity was "50 times better than that available to Podkletnov." [Quoting from Hathaway 2003].

\section{Coronal Blowers}

There are many variants of the original patent where high-voltage capacitors create thrust [Brown 1928], many of which claim that the thrust is a new physical affect akin to antigravity. These go by such terms as: "Biefeld-Brown effect," "lifters," "electrostatic antigravity," "electrogravitics," and "asymmetrical capacitors." To date, all rigorous experimental tests indicate that the observed thrust is attributable to coronal wind [Canning 2004, Tajmar 2004, Talley 1991]. Quoting from one such finding: "... their operation is fully explained by a very simple theory that uses only electrostatic forces and the transfer of momentum by multiple collisions [with air molecules]." [Canning 2004]

\section{Quantum Tunneling as an FTL venue}

A prerequisite to faster-than-light travel is to prove faster-than-light information transfer. The phenomenon of quantum tunneling, where signals appear to pass through barriers at superluminal speed, is often cited as such empirical evidence. Experimental and theoretical work indicates that the information transfer rate is only apparently superluminal, with no causality violations. Although the leading edge of the signal does appear to make it through the barrier faster, the entire signal is still light-speed limited [Mojahedi 2000a, 2000b, and Segev 2000]. This topic still serves, however, as a tool to explore this intriguing aspect of physics.

\section{Unresolved Approaches}

\section{Woodward's Transient Inertial Oscillations}

Experiments and theories published by James Woodward claim that oscillatory changes to inertia can be induced by electromagnetic means [Woodward 2004] and a patent exists on how this can be used for propulsion [Woodward 1994]. Conservation of momentum is satisfied by evoking interpretations of Mach's principle. Independent verification experiments, using techniques less prone to spurious effects, were unable to reliably confirm or dismiss the claims [Cramer 2004]. Woodward and others continue with 
experiments and publications to make the effect more pronounced and to more clearly separate the claimed effects from experimental artifacts. This oscillatory inertia approach is considered unresolved.

\section{Abraham-Minkowski Electromagnetic Momentum}

More than one approach attempts to use an unresolved question of electromagnetic momentum (Abraham-Minkowski controversy [Brevik 1982]) to suggest a new space propulsion method [Slepian 1949, Brito 2001, and Corum 2001]. The equations that describe electromagnetic momentum in vacuum are well established (photon radiation pressure), but there is still debate concerning momentum within dielectric media. In all of the proposed propulsion methods, the anticipated forces are relatively small (comparable to experimental noise) and critical issues remain unresolved. In particular, the conversion of an oscillatory force into a net force remains questionable and the issue of generating external forces from different internal momenta remains unproven. Even if unsuitable for propulsion, these approaches provide empirical tools for further exploring the Abraham-Minkowski controversy of electromagnetic momentum.

\section{Inertia and Gravity Interpreted as Quantum Vacuum Effects}

Theories are entering the peer-reviewed literature that assert that gravity and inertia are side effects of the quantum vacuum. The theories are controversial and face many unresolved issues. In essence this approach asserts that inertia is related to an electromagnetic drag force against the vacuum when matter is accelerated, and that gravity is the result of asymmetric distributions of vacuum energy caused by the presence of matter [Puthoff 1993, Haisch 1994, Rueda 1998, and Puthoff 2005]. The space propulsion implications of these theories have been raised [Puthoff 2002], but experimental approaches to test these assertions have not yet entered the literature.

\section{Podkletnov Force Beam}

On an Internet physics archive [Pokkletnov 2001] it is claimed that forces can be imparted to distant objects using high-voltage electrical discharges near superconductors. Between $4 \times 10^{-4}$ to $23 \times 10^{-4}$ Joules of mechanical energy are claimed to have been imparted to an 18.5-gram pendulum located 150 meters away and behind brick walls of a separate building. Like the prior gravity shielding claims, these experiments are difficult and costly to duplicate, and remain unsubstantiated by reliable independent sources.

\section{Candidates for Continued Research}

\section{Space Drives}

"Space drive" is a general term to encompass the ambition of propulsion without propellant. To identify the unresolved issues and research paths toward creating a space drive, seven hypothetical space drives were conceived and cursorily addressed [Millis 1997]. The two largest issues facing this ambition are to find a way for a vehicle to induce external net forces on itself, and secondly, to satisfy conservation of momentum in the process. As discussed below, several avenues for research remain, including: (1) investigate space from the perspective of new sources of reaction mass, (2) revisit Mach's Principle to consider coupling to surrounding mass via inertial frames, and (3) investigate the coupling between gravity, inertia, and controllable electromagnetic phenomena. These are very broad and open areas where a variety of research sequels could emerge.

Reaction mass in space.-A key aspect of conservation of momentum is the reaction mass. When an automobile accelerates, its wheels push against the road using the Earth as the reaction mass. Helicopters and aircraft use the air as their reaction mass. In space, where there are no roads or air, a rocket must 
bring along propellant to thrust against. To contemplate space travel that circumvents the propellant limits of rockets, some other indigenous reaction mass must be found along with the means to induce net forces on the reaction mass.

Recent observations reveal a number of interesting phenomena of space. Although none are directly suitable as reactive media, they are at least indicative that space has substantive properties whose further study pertains to breakthrough spaceflight. Cosmological observations have revealed the Cosmic Microwave Background Radiation, dark matter, and dark energy [Hartle 2002], and quantum physics has revealed zero point energy [Milloni 1994]. The Cosmic Background Radiation is low-energy microwave radiation whose composite motion is coincident with the mean reference frame of the universe [Muller 1978]. Although too weak to be used as a reactive media, its existence and directional dependence is thought provoking in the context of space travel. Dark matter is the term used to encompass observations that there is more gravitating matter at galactic scales than can be observed. Some estimates are that more than 90 percent of the matter in galaxies is not directly visible. One of the key supporting empirical observations are the anomalous rotation rates of galaxies, where the galaxies appear to hold together more strongly than can be accounted for by the visible matter. From the propulsion point of view, the suitability of dark matter as a reaction mass has not yet been rigorously studied. On even larger scales, anomalous red-shifts from the most distant matter of the universe suggest that the universal expanding at an accelerating rate. The working hypothesis for this anomaly is dubbed dark energy and it is conjectured to be an antigravity-like effect [Seife 2003]. Again, the propulsion implications of such phenomena have not been explored. And lastly, the quantum vacuum phenomenon of zero point energy suggests that even the most empty of spaces still contain some non-zero amount of energy. This last item is discussed separately in a later paragraph.

Revisit Mach's Principle. - One of the theoretical approaches in dealing with momentum conservation for space drives is to reexamine Mach's Principle. Mach's Principle asserts that an inertial frame, specifically the property of a space to be a reference frame for acceleration, is actually created by and connected to the surrounding mass in the universe [Mach 1883]. At least one perspective views this property as being related to the gravitational potential of the masses across the universe [Barbour 1995]. A related issue is that a literal interpretation of Mach's Principle implies an absolute reference frame, coincident with the mean rest frame for all the matter in the universe [Barbour 1989]. From the spacepropulsion point of view, this is a convenient perspective. Curiously, a known phenomenon that is coincident with this reference frame is the Cosmic Microwave Background Radiation.

These Machian perspectives imply a Euclidean view of space-time. Within general relativity, there do exist such Euclidean interpretations, which are often referred to as "optical analogies." In this interpretation, space is represented as an optical medium with an effective index of refraction that is a function gravitational potential [de Felice 1971, Evans 1996]. Although different from the more common geometric interpretation, this interpretation has been shown to be consistent with physical observables, and transformation rules between the optical and geometric perspectives have also been published [Evans 1996]. Conveniently, it also casts the coupling between gravity and electromagnetism in more simple terms. Little attention is typically focused on this optical analogy because it does not predict any new effects that aren't already covered by the more common geometric perspective and because it raises unanswered issues with coordinate systems choices. Another consequence is that wormholes are indescribable in this perspective. From the propulsion point of view, however, issues of coordinate frames are of keen interest.

Coupling of fundamental forces. - Electromagnetism, gravity and spacetime are coupled phenomena. Given our technical proficiency at manipulating electromagnetism, this coupling hints that we might be able to use electromagnetism to affect gravity. In principle this is true. In practice, at least from the perspective of general relativity, it would take an enormous amount of electromagnetic energy to produce a perceptible gravitational effect - energy levels in the regime of $\mathrm{E}=\mathrm{mc}^{2}$, where $\mathrm{m}$ represents the induced mass effect. While general relativity pertains to large-scale couplings, quantum and particle physics pertains to the couplings on the atomic scale and smaller. One example of an unresolved smallscale question is the unknown inertial and gravitational properties of antimatter. Although presumed to be 
equal to their normal-matter counterparts, long-duration low-gravity experiments could resolve minor differences that have not been testable in terrestrial labs [Lewis 1998]. Such experiments might also help resolve the lingering incompatibility between general relativity and quantum mechanics. As much as these pertain to general physics, they may also have implications for propulsion physics.

\section{Quantum Vacuum Energy Experiments}

The uncertainty principle from quantum mechanics indicates that it is impossible to achieve an absolute zero energy state. This includes the energy state of empty space [Milonni 1994]. It has been shown analytically [Casimir 1948], and later experimentally [Lamoreaux 1997], that this vacuum energy can squeeze parallel plates together. This "Casimir effect" is only appreciable for very small cavity dimensions (microns). Nonetheless, it is evidence that empty space can present situations where forces exist when none were naively expected. Theoretically it might be possible to induce net forces relative to this background energy, but the forces are extremely small [Maclay 2004]. More recent experiments have explored the physics of the quantum vacuum using MEMS technology-micro-electro-mechanical structures of machined silicon [Maclay 2000, Esquivel-Sirvent 2002]. Continued research on this phenomenon and through these techniques is expected.

\section{Provocative Questions}

In addition to the unanswered questions of reaction mass in space or the viability of vacuum energy for practical purposes, there are a variety of other provocative effects and theoretical questions that pertain to the search for new propulsion physics. One example from general relativity is that a propulsive effect could be induced by frame dragging from a twisting toroid of ultra dense matter, where an acceleration field is induced inside the toroid [Forward 1963]. Although the magnitude of the induced effect is trivial compared to the energy expenditure, this serves as an analytical approach to investigate the implications of such notions. Another curiosity is the anomalous trajectories of the Pioneer 10/11, Galileo, and Ulysses spacecraft [Anderson 1998, 1999]. Once these spacecraft were farther than about 20 astronomical units from the Sun, their actual trajectories show an unexpected deceleration on the order of $10^{-10} \mathrm{~g}$ 's [Nieto 2004]. A report sponsored by the European Space Agency includes a proposal for a "Sputnik-5" probe to explore this anomaly [Bertolami 2002]. This same ESA study further suggests checking for evidence of violations of the equivalence principle in long duration free-fall trajectories (orbits).

\section{Faster Than Light}

As a consequence of Einstein's general relativity, the notion of warping space to circumvent the lightspeed limit is an open topic in scientific literature. This approach involves altering spacetime itself rather than trying to break the light-speed limit through spacetime. Two prominent approaches are the warp drive and the wormhole. The warp drive concept involves moving a bubble of spacetime that carries a vehicle within [Alcubierre 1994]. A wormhole, on the other hand, is a shortcut through spacetime created by extreme spacetime warping [Morris 1998, Visser 1995]. Enormous technical hurdles face these concepts. In particular, they require enormous quantities of "negative energy" (equivalent mass of planets or suns) [Pfenning 1998], and evoke time-travel paradoxes ("closed-time-like curves") [Everett 1996]. Given the magnitude of energy requirements to create perceptible effects, it is unlikely that experimental work will be forthcoming in the near future. Even though these theoretical concepts are unlikely to be engineered, they are at least useful for teaching the intricacies of general relativity. While laboratory experiments are still prohibitive, astronomical searches for related phenomena could be undertaken, such as looking for the characteristic signatures of a wormhole [Cramer 1995]. 


\section{Summary of Research Findings}

The majority of open research paths involve further study of the fundamental properties of spacetime and inertial frames, looking for candidate sources of reaction mass and the means to interact with it. As much as these are basic areas of investigation for general physics, their investigation in the context of breakthrough spaceflight introduces additional perspectives from which to contemplate these lingering unknowns. This alternative perspective might just provide the insight that would otherwise be overlooked.

\section{Concluding Remarks}

The potential benefits of breakthrough propulsion cannot yet be calculated with certainty, but crude introductory assessments show that the performance gains could span from a factor of 2 to a factor of $10^{150}$ improvement in the amount of energy required to move an object from one point to another. The more demanding the journey, the higher the gain. For a hypothetical non-relativistic space drive, the energy scales as the square of the $\Delta v$, while rocket energy scales exponentially for $\Delta v$. This is a considerable difference, particularly for high $\Delta v$ missions..

Because of the profound implications of success and the fledgling nature of the research, special management methods are recommended to ensure credible progress. Lessons from the NASA Breakthroughs Propulsion Physics Project include: (1) constraining the research tasks to only address immediate unknowns, curious effects or critical issues, (2) putting more emphasis on the reliability of assertions than their implications, and (3) having reviewers judge credibility rather than feasibility.

The search for breakthrough propulsion methods is an embryonic field encompassing many differing approaches and challenges. The majority of open research paths involve further study of possible reaction masses in space, the physics of inertial frames, the properties of the quantum vacuum, and the coupling of electromagnetism, spacetime and gravity. As much as these are basic areas of investigation for general physics, their investigation in the context of breakthrough spaceflight introduces another perspective from which to contemplate these lingering unknowns. This alternative perspective might just provide an insight that would otherwise be overlooked.

Much of the research is conducted as individual discretionary efforts, scattered across various government, academic, and private organizations. In addition to the research already described, there are many more approaches emerging in the literature and at aerospace conferences. At this stage it is still too early to predict which, if any, of the approaches might lead to a breakthrough. Taken objectively, the desired breakthroughs might also remain impossible to achieve. Reciprocally, however, history has shown that breakthroughs tend to take the pessimists by surprise.

\section{References}

Alcubierre, M. (1994), “The warp drive: hyper-fast travel within general relativity," Class. Quantum Gravity, 11, p. L73.

Anderson, J.D., et al. (1998), "Indication from Pioneer 10/11, Galileo and Ulysses Data of an Apparent Anomalous Weak Long-range Acceleration," Phys Rev Let, 81, pp. 2858-2861; and response to comments in Phys Rev Let, 83 (1999), p. 1891.

Barbour, J., and Pfister, H., eds. (1995), Mach's Principle: From Newton's Bucket to Quantum Gravity, Birkhauser, Boston.

Barbour, J. (1989) Absolute or relative motion?: a study from Machian point of view of the discovery and the structure of dynamical theories, Cambridge University Press, Cambridge, England; New York.

Berman, A.I., (1961) The Physical Principles of Astronautics: Fundamentals of Dynamical Astronomy and Space Flight, John Wiley \& Sons, NY, p.247.

Bertolami, O., and Tajmar (2002), "Gravity Control and Possible Influence on Space Propulsion: A Scientific Study,” ESA CR (P) 4365, on Contract ESTEC 15464/01/NL/Sfe. 
Boston, M. (2000), "Simplistic Propulsion Analysis of a Breakthrough Space Drive for Voyager," AIP Conf. Proc. No. 504, El-Genk, ed., American Institute of Physics, New York, NY, pp. 1075-1078

Brevik, I. (1982) Comment on 'Electromagnetic Momentum in Static Fields and the Abraham-Minkowski Controversy, Physics Letters 88 A, pp. 335-338.

Brito, H.H., (2001), "Experimental Status of Thrusting by Electromagnetic Inertia Manipulation," Paper IAF-01-S.6.02, 52nd International Astronautical Congress, Toulouse France.

Brown, T.T. (1928), "A Method of and an Apparatus or Machine for Producing Force or Motion," GB Patent \#300,311.

Byers, D.C. (1969), “An Experimental Investigation of a High Voltage Electron Bombardment Ion Thruster," J. of the Electrochemical Society, 116(1), pp. 9-17.

Canning, F.X., Melcher, C. \& Winet, E. (2004), “ Asymmetrical Capacitors for Propulsion,” NASA CR2004-213312.

Casimir, H.B.G., and Polder, D. (1948), "The Influence of Retardation on the London-van der Walls Forces," Physical Review, 73(4), p. 360-372.

Corum, J.F., Keech, Kapin, Gray, Pesavento, Duncan, and Spadaro (2001), "The Electromagnetic StressTensor as a Possible Space Drive Propulsion Concept,” AIAA-2001-3654, Joint Propulsion Conference, Salt Lake City, UT.

Cramer, J., Forward, R.L., Morris, M., Visser, M., Benford, G. and Landis, G. (1995), "Natural Wormholes as Gravitational Lenses," Physical Review D, (March 15, 1995), pp. 3124-3127.

Cramer, J., Fey, C.W., and Casissi, D.V. (2004), "Tests of Mach's Principle with a Mechanical Oscillator," NASA/CR-2004-213310.

de Felice (1971), "On the Gravitational Field Acting as an Optical Medium," General Relativity and Gravitation, 2, pp. 347-357.

Esquivel-Sirvent, R., Villarreal, C., Mochan, W.L. and Cocoletzi, G.H., (2002)"Casimir Forces in Nanostructures," Physica Status Solidi (b), 230, p. 409.

Evans, J., Nandi, and Islam (1996), "The Optical-Mechanical Analogy in General Relativity: Exact Newtonian Forms for the Equations of Motion of Particles and Photons," General Relativity and Gravitation, 28, pp. 413-439.

Everett, A.E., (1996), Warp drive and causality, Phys. Rev. D 53, 7365-7368 (15 June 1996).

Forward, R.L. (1963), "Guidelines to Antigravity," American Journal of Physics, 31, pp. 166-170.

Foster, R.N. (1986), Innovation; The Attacker's Advantage, Summit Books.

Fralick G. and Niedra (2001), "Experimental Results of Schlicher's Thrusting Antenna," AIAA-20013657, Joint Propulsion Conference, Salt Lake City, UT.

Haisch, B., Rueda, and Puthoff, "Inertia as a zero-point field Lorentz force," Phys. Rev. A, 49, p. 678.

Hartle, J.B. (2002), Gravity: An Introduction to Einstein's General Relativity, Addison Wesley; 1st ed.

Hathaway, Cleveland, and Bao (2003), "Gravity modification experiment using a rotating superconducting disk and radio frequency fields," Physica C, 385, pp. 488-500.

Hord, R.M. (1985), CRC Handbook of Space Technology: Status and Projections, CRC Press, Boca Raton, FL.

Krasnikov, S.V. (1998), "Hyperfast Interstellar Travel in General Relativity," Phys. Rev. D, 57, p. 4760.

Lewis, R.A., Smith, G.A., Huber, F.M., and Messerschmid, E.W. (1998), "Precision Tests of Einstein's Weak Equivalence Principle for Antimatter," AIP Conference Proceedings, 420, STAIF, Albuquerque, NN, pp. 1435-1442.

Mach, E. (1883), The Science of Mechanics, Fifth English Edition, Open Court Publishing Co, London, (1942).

Maclay, G.J., (2000) "Analysis of zero-point electromagnetic energy and Casimir forces in conducting rectangular cavities," Physical Review A, 61, 052110-1 to 052110-18.

Maclay, J. and Forward, R. (2004), "A Gedanken spacecraft that operates using the quantum vacuum (adiabatic Casimir effect)," Foundations of Physics, 34(3), pp. 477-500. 
Millis, M.G., and Williamson, G.S. (1995), Experimental Results of Hooper's Gravity-Electromagnetic Coupling Concept, NASA TM-106963, AIAA-95-2601, 31st Joint Propulsion Conference, San Diego CA.

Millis, M.G. (1997), "Challenge to Create the Space Drive," AIAA Journal of Propulsion and Power, 13(5), pp. 577-582.

Millis, M.G. (1999a), "NASA Breakthrough Propulsion Physics Program,” Acta Astronautica, 44, Nos. 2-4, pp. 175-182.

Millis, M.G., (2004a), "Prospects of Breakthrough Propulsion from Physics," NASA TM-2004-213082.

Millis, M.G., (2004b), "Breakthrough Propulsion Physics Project: Project Management Methods," NASA TM-2004-213406.

Milonni, P.W. (1994), The Quantum Vacuum, Academic Press, San Diego, CA.

Misner C.W., Thorne, K.W., and Wheeler, J.A. (1973), Gravitation, W. H. Freeman \& Company, NY.

Mojahedi, M., Schamiloglu, E., Hegeler, F., and Malloy, K. J. (2000a), "Time-domain detection of superluminal group velocity for single microwave pulses," Physical Review E, 62, pp. 5758-5766.

Mojahedi, M., Schamiloglu, E. Kamil, A. and Malloy, K. J. (2000b), "Frequency and Time-Domain Detection of Superluminal Group Velocities in a Distributed Bragg Reflector," IEEE Journal of Quantum Electronics, 36, pp. 418-424.

Morris, M.S., Thorne, and Yurtsever (1988), "Wormholes, time machines, and the weak energy condition," Physical Review Letters, 61, p. 1446.

Morris, M.S., and Thorne (1988), "Wormholes in spacetime and their use for interstellar travel: A tool for teaching general relativity," American Journal of Physics, 56, pp. 395-412.

Muller, R. (1978), "The Cosmic Background Radiation and the new Aether Drift," Scientific American, 238, pp. 64-74.

Nieto, M.M. and Turyshev, S.G. (2004), Finding the origin of the Pioneer anomaly, Class. Quantum Grav. 21 4005-4023

Pfenning, M.J. and Ford L.H. (1998), Quantum inequality restrictions on negative energy densities in curved spacetimes, Doctoral dissertation, Tufts University Arxiv preprint gr-qc/9805037, 1998 arxiv.org

Podkletnov E. and Nieminen (1992), "A Possibility of Gravitational Force Shielding by Bulk YBCO Superconductor," Physica C, 203, pp. 441-444.

Podkletnov, E., and Modanese (2001), "Impulse Gravity Generator Based on Charged YBa2Cu3O7-y Superconductor with Composite Crystal Structure," arXiv:physics/ 0108005 v2.

Puthoff, Little and Ibison (2002), "Engineering the zero-point field and polarizable vacuum for interstellar flight," Jour. Brit. Interplanetary Soc. (JBIS), 55, pp. 137-144.

Puthoff, H.E. (1993), "Gravity as a zero-point-fluctuation force," Phys. Rev. A, 39, p. 2333; Comments, Phys. Rev A, 47, p.3454.

Puthoff, H.E. (2002), "Polarizable-Vacuum (PV) approach to general relativity," Found. Phys., 32, pp. 927-943.

Puthoff, H.E., Davis, E.W., and Maccone, C. (2005), "Levi-Civita effect in the polarizable vacuum (PV) representation of general relativity," Gen. Relativity \& Gravity, 37(3), pp. 483-489.

Rueda and Haisch (1998), "Inertial mass as reaction of the vacuum to accelerated motion," Phys. Letters $A, \mathbf{2 4 0}$, pp. 115-126.

Resnick, R. and Halliday, D., (1977) Physics, 3rd ed., John Wiley \& Sons, NY.

Schlicher, R.L, Biggs, and Tedeschi, "Mechanical Propulsion from Unsymmetrical Magnetic induction Fields," AIAA 95-2643, Joint Propulsion Conference, San Diego, CA (1995).

Segev, Bilha, Milonni, Peter W., Babb, James F., and Chiao, Raymond Y., (2000)"Quantum noise and superluminal propagation," Physical Review A, 62, 0022114-1 - 0022114-15.

Seife, C., "Dark Energy Tiptoes Toward the Spotlight," Science, 300 (5627), pp. 1896-1897 (20 June 2003)

Seifert, H. ed, (1959) Space Technology (based on a University of California engineering extension course), John Wiley \& Sons, NY. 
Slepian, J., "Electromagnetic Space-Ship,” Electrical Engineering, pp. 145-146 (March 1949).

Sparnaay, M.J., "Measurements of Attractive Forces between Flat Plates," Physica, 24 (1958), pp. 751-764.

Speake, C.C., and Trenkel, "Forces between Conducting Surfaces due to Spatial Variations of Surface Potential," Physical Review Letters, No. 160403 (25 April 2003).

Tajmar, M., "The Biefeld-Brown Effect: Misinterpretation of Corona Wind Phenomena," AIAA Journal of Propulsion \& Power, 42, (2004), pp. 315-318.

Tajmar, M. and Bertolami, O. (2005), "Hypothetical Gravity Control and Possible Influence on Space Propulsion," AIAA Journal of Propulsion and Power, 21, pp. 692-696, (2005) http://www.arxiv.org/abs/physics/0412176

Talley, R.L. (1991), “Twenty First Century Propulsion Concept,” PL-TR-91-3009, Final Report for the period Feb 89 to July 90, on Contract FO4611-89-C-0023, Phillips Laboratory, Air Force Systems Command, Edwards AFB, CA.

Thomas, N. (2002), "Common Errors," on the NASA Breakthrough Propulsion Physics Project web site: http://www.grc.nasa.gov/WWW/bpp/ComnErr.html

Visser, M. (1995), Lorentzian Wormholes: from Einstein to Hawking, AIP Press, New York.

Woodward, J.F (1994), "Method for Transiently Altering the Mass of an Object to Facilitate Their Transport or Change their Stationary Apparent Weights," US Patent \# 5,280,864.

Woodward, J.F. (2004), "Flux Capacitors and the Origin of Inertia," Foundations of Physics 34, pp. $1475-1514$. 


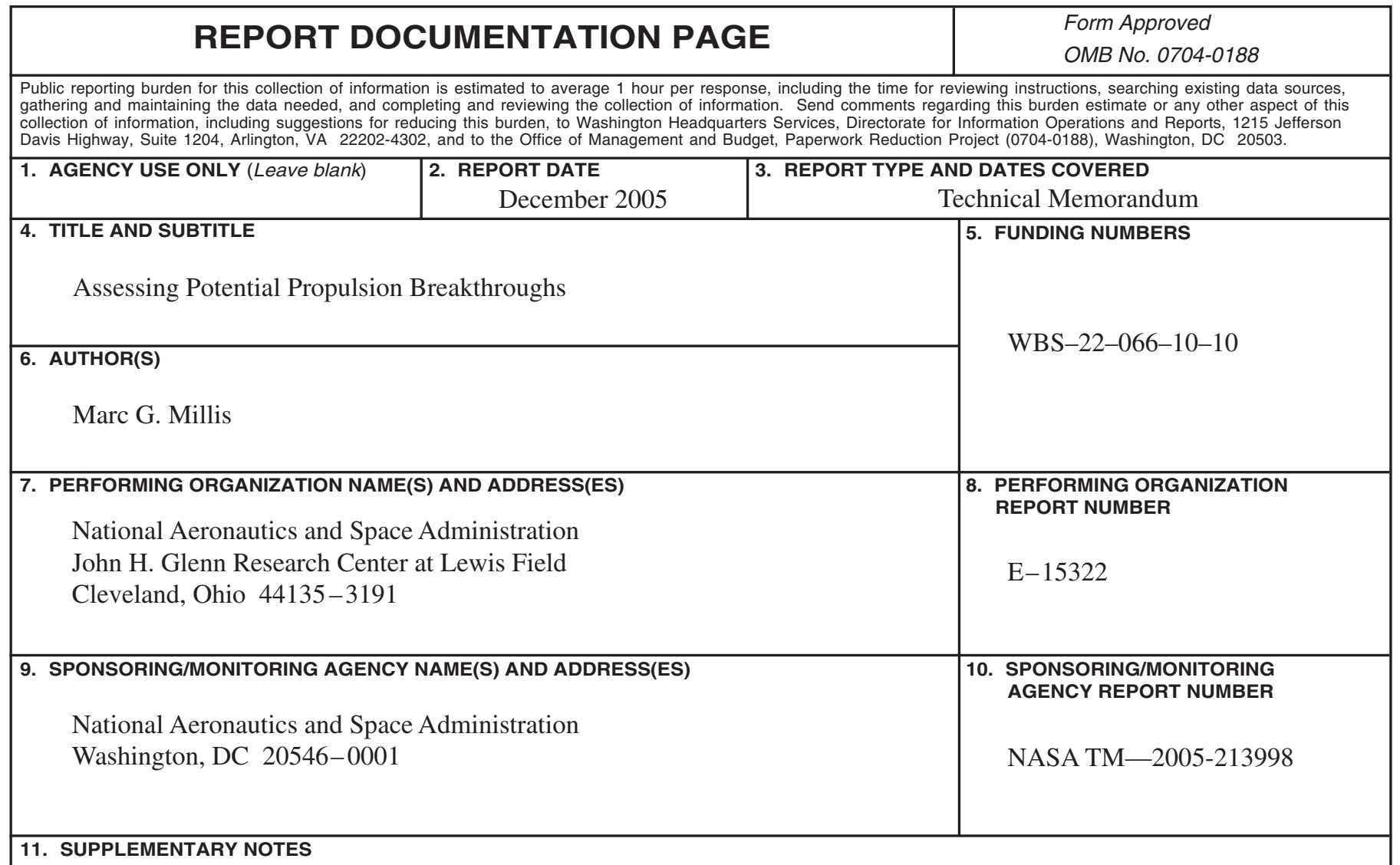

Prepared for the New Trends in Astrodynamics and Applications II-An International Conference sponsored by NASA Headquarters and Princeton University, Princeton, New Jersey, June 3-5, 2005. Responsible person, Marc G. Millis, organization code RTP, 216-977-7535.

12a. DISTRIBUTION/AVAILABILITY STATEMENT 12b. DISTRIBUTION CODE

Unclassified - Unlimited

Subject Categories: 70, 20, and 44

Available electronically at http://gltrs.grc.nasa.gov

This publication is available from the NASA Center for AeroSpace Information, 301-621-0390.

13. ABSTRACT (Maximum 200 words)

The term, propulsion breakthrough, refers to concepts like propellantless space drives and faster-than-light travel, the kind of breakthroughs that would make interstellar exploration practical. Although no such breakthroughs appear imminent, a variety of investigations into these goals have begun. From 1996 to 2002, NASA supported the Breakthrough Propulsion Physics Project to examine physics in the context of breakthrough spaceflight. Three facets of these assessments are now reported: predicting benefits, selecting research, and recent technical progress. Predicting benefits is challenging since the breakthroughs are still only notional concepts, but kinetic energy can serve as a basis for comparison. In terms of kinetic energy, a hypothetical space drive could require many orders of magnitude less energy than a rocket for journeys to our nearest neighboring star. Assessing research options is challenging when the goals are beyond known physics and when the implications of success are profound. To mitigate the challenges, a selection process is described where: research tasks are constrained to only address the immediate unknowns, curious effects or critical issues; reliability of assertions is more important than their implications; and reviewers judge credibility rather than feasibility. The recent findings of a number of tasks, some selected using this process, are discussed. Of the 14 tasks included, 6 reached null conclusions, 4 remain unresolved, and 4 have opportunities for sequels. A dominant theme with the sequels is research about the properties of space, inertial frames, and the quantum vacuum. 14. SUBJECT TERMS

Spacecraft propulsion; Physics; Project management; Relativity; Antigravity

\begin{tabular}{|c|c|c|}
\hline $\begin{array}{c}\text { 17. SECURITY CLASSIFICATION } \\
\text { OF REPORT } \\
\text { Unclassified }\end{array}$ & $\begin{array}{c}\text { 18. SECURITY CLASSIFICATION } \\
\text { OF THIS PAGE } \\
\text { Unclassified }\end{array}$ & $\begin{array}{c}\text { 19. SECURITY CLASSIFICATION } \\
\text { OF ABSTRACT } \\
\text { Unclassified }\end{array}$ \\
\hline
\end{tabular}

NSN 7540-01-280-5500

29

15. NUMBER OF PAGES

16. PRICE CODE

20. LIMITATION OF ABSTRACT

Standard Form 298 (Rev. 2-89)

Prescribed by ANSI Std. Z39-18 298-102 

